\title{
BALANÇO DE ENERGIA E ESTIMATIVA DA EVAPOTRANSPIRAÇÃO EM CULTURAS IRRIGADAS DE MARACUJAZEIRO PELO MÉTODO DA RAZÃO DE BOWEN
}

\author{
TONNY J. A. SILVA ${ }^{1}$, MARCOS V. FOLEGATTI ${ }^{2}$, CLÁUDIO R. SILVA ${ }^{1}$, \\ JOSÉ ALVES JÚNIOR ${ }^{1}$, EDNA M. BONFIM-SILVA ${ }^{3}$
}

\begin{abstract}
RESUMO: A cultura do maracujazeiro-amarelo tem tido grande expansão da área cultivada no Estado de São Paulo e, apesar da importância da irrigação para a cultura, há poucos estudos sobre sua utilização. Este trabalho foi realizado na área experimental de irrigação, na Fazenda Areão, do Departamento de Engenharia Rural, da Escola Superior de Agricultura "Luiz de Queiroz" ESALQ/USP (Piracicaba - SP). Foram coletados dados de saldo de radiação, fluxo de calor no solo, gradientes de temperatura e pressão de vapor do ar com o objetivo de avaliar a partição da energia disponível e a evapotranspiração em cultivo de maracujazeiros em dois sistemas de plantio (linhas de cultivo norte-sul e leste-oeste). O método da razão de Bowen foi utilizado na determinação dos fluxos de calor sensível e latente. O saldo de radiação não foi influenciado pelo sistema de plantio, sendo equivalente a $60 \%$ da radiação global. O componente de maior contribuição no balanço de energia foi o calor latente de evaporação ocorrendo imediatamente após a chuva ou irrigação. A utilização do modelo de análise e exclusão de intervalos em que o método da Razão de Bowen falha, melhora a estimativa da evapotranspiração.
\end{abstract}

PALAVRAS-CHAVE: irrigação, fluxo de calor latente e sensível, micrometeorologia.

\section{ENERGY BALANCE AND ESTIMATIVE OF THE EVAPOTRANSPIRATION OF IRRIGATED PASSIONFRUIT CROPS BY THE BOWEN RATIO METHOD}

\begin{abstract}
Passionfruit crop has been having great expansion in the cultivated area of São Paulo State and beside the irrigation importance for crop; there are few studies about its utilization. This work was carried out at the experimental irrigation farm of the Engineering Department College of Agriculture "Luiz de Queiroz" - ESALQ/USP, in Piracicaba, São Paulo State, Brazil. Were collected micrometeorological data from net radiation, soil flux heat, temperature and vapor pressure gradient with the aim to evaluate the partition of available energy and evapotranspiration of passionfruit crop planted under two row directions (North-South and East-West). The Bowen ratio method was used to determine both sensible and latent fluxes. The net radiation was not influenced by row directions, being $60 \%$ of global radiation. The latent heat of evaporation played the major role on energy balance and values more significantly happened immediately after a rainfall or irrigation. The estimative of evapotranspiration has improved when the interval that Bowen ratio fails were excluded.
\end{abstract}

KEYWORDS: irrigation, latent and sensible heat fluxes, micrometeoroly.

\footnotetext{
${ }^{1}$ Eng ${ }^{0}$ Agrônomo, Doutor em Irrigação e Drenagem pela Escola Superior de Agricultura “Luiz de Queiroz” ESALQ-USP.

${ }^{2}$ Eng ${ }^{\mathrm{o}}$ Agrônomo, Prof. Doutor, Departamento de Engenharia Rural, ESALQ/USP, Piracicaba - SP.

${ }^{3}$ Pós-Doutora em Solos e Nutrição de Plantas, ESALQ/USP, Piracicaba - SP.

Recebido pelo Conselho Editorial em: 26-4-2005

Aprovado pelo Conselho Editorial em: 2-6-2007
} 


\section{INTRODUÇÃO}

O maracujazeiro-amarelo (Passiflora edulis Sins var. flavicarpa Deg) é a principal espécie de importância econômica da família Passifloraceae. O Estado de São Paulo aparece com a maior expansão de área cultivada, onde a cultura é bem adaptada. Trata-se de atividade bastante atrativa para pequenos produtores, uma vez que oferece retorno econômico rápido, com receitas distribuídas quase o ano inteiro (SOUZA \& MELLETI, 1997), principalmente quando se adota a irrigação.

O conhecimento da evapotranspiração torna-se necessário para o manejo de irrigação. A temperatura, a radiação, o fotoperíodo, a precipitação e o vento são os elementos meteorológicos que condicionam a evapotranspiração (PEREIRA et al., 2002). Constata-se, ainda, a existência de poucos estudos sobre parâmetros micrometeorológicos que atuam na evapotranspiração da cultura do maracujazeiro.

O processo de evapotranspiração é governado pela troca de energia na superfície, limitada pela energia disponível. É possível estimar o fluxo de calor latente de evaporação por meio do princípio da conservação de energia: $R n=G+H+\lambda E$ (TEIXEIRA, 2001), e a evapotranspiração por meio da equação simplificada do balanço de energia, constituída pelo saldo de radiação $(R n)$, pelo fluxo de calor latente $(\lambda E)$, calor sensível $(H)$ e pelo fluxo de calor no solo $(G)$. A solução da equação é obtida por medidas de $R n$ e $G$ e de estimativas de $\lambda E$ e $H$, utilizando o método da Razão de Bowen (BOWEN, 1926), que considera a relação $\beta=H / \lambda E$.

Em muitos trabalhos em que o método de Razão de Bowen-Balanço de Radiação $(R B B R)$ é utilizado, para evitar erros na estimativa dos fluxos, os dados inerentes ao erro instrumental micrometeorológico do sistema Razão de Bowen são excluídos. Um exemplo é o caso de os intervalos de valores dos gradientes de $H, \Delta T$ e pressão de vapor serem menores do que as resoluções dos sensores. Por outro lado, para casos em que os valores de $\beta$ estão próximo de -1 , alguns autores eliminam os valores de $\beta$ abaixo de $-0,75$ ou valores entre $-1,3<\beta<-0,7$ (ORTEGA-FARIAS et al., 1996; UNLAND et al., 1996).

PEREZ et al. (1999) propõem critérios para determinar os valores confiáveis de $\beta$ e de fluxos de calor latente $(\lambda E)$ e sensível $(H)$ baseados nos limites de resolução dos sensores e gradientes de pressão de vapor, fornecendo resposta prática e clara ao problema da avaliação de quando o método de $R B B R$ fornece valores inconsistentes.

O objetivo do presente estudo foi quantificar a partição da energia solar disponível e a evapotranspiração de maracujazeiros em duas áreas com diferentes orientações de plantio (linhas de cultivo norte-sul e leste-oeste), empregando-se o método de Razão de Bowen e avaliar um método de identificação e exclusão de dados inconsistentes da Razão de Bowen.

\section{MATERIAL E MÉTODOS}

\section{Localização e descrição do sistema de cultivo}

O experimento foi conduzido na área experimental de irrigação, na Fazenda Areão, do Departamento de Engenharia Rural, da Escola Superior de Agricultura "Luiz de Queiroz" -

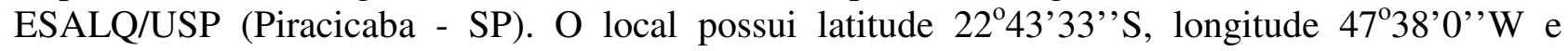
altitude de $576 \mathrm{~m}$. O clima é do tipo Cwa, segundo a classificação climática de Köeppen, sendo subtropical úmido com estiagem no inverno, temperatura e precipitação médias anuais de $21,1{ }^{\circ} \mathrm{C} \mathrm{e}$ $1.250 \mathrm{~mm}$, respectivamente. O solo foi classificado como Nitossolo (EMBRAPA, 1999).

O transplantio de mudas de maracujazeiro (Passiflora edulis, cultivar IAC 275) foi realizado em 25-10-2003, aos 83 dias após a germinação. O sistema de condução foi do tipo espaldeira vertical, com espaçamento entre mourões e entre plantas de $4 \mathrm{~m}$ x $4 \mathrm{~m}$, com um único fio de arame a 2 m acima da superfície do terreno. 
Toda a área (aproximadamente 0,5 ha) foi irrigada por microaspersão, procurando manter o potencial de água no solo acima do limite de $-20 \mathrm{kPa}$, monitorado por seis tensiômetros (duas repetições de três tensiômetros), à profundidade de $0,10 \mathrm{~m}$. O volume total de água aplicado (evapotranspiração da cultura) foi determinado pela média das leituras diárias de dois lisímetros de pesagem hidráulica (de $1 \mathrm{~m}^{2}$ de área), cultivado com um maracujazeiro cada, instalados no centro da área experimental. O tempo de irrigação foi estabelecido de acordo com a vazão do microaspersor $\left(40 \mathrm{~L} \mathrm{~h}^{-1}\right)$, sendo um microaspersor por planta.

\section{Cálculo da partição de energia e evapotranspiração}

Foram realizadas medidas de $R n, G$ e dos gradientes de temperatura do ar $(\Delta T)$, calculada a pressão atual de vapor $\left(\Delta e_{a}\right)$ a $0,2 \mathrm{~m}$ e a $1,2 \mathrm{~m}$, dois níveis acima do dossel da cultura (que era, aproximadamente, $2 \mathrm{~m}$ ). Segundo OMETTO (1981), a teoria de Austausch estabelece que os valores representativos de gradientes são obtidos quando os psicrômetros estejam instalados o mais próximo possível da cobertura vegetal, estando o psicrômetro inferior aproximadamente a 0,10,2 m do nível do dossel, e o superior obedecendo a essas distâncias $(0,1-0,2 \mathrm{~m})$ em relação ao inferior. Com as medidas de $R n, G$, e $\Delta T$, foram calculados os fluxos $\lambda E$ [eq.(1)] e $H$ [eq.(2)], empregando-se a eq.(3) (KUSTAS et al., 1996), segundo a razão de Bowen [eq.(4)], pelo princípio da conservação de energia.

$$
\begin{aligned}
& \lambda E=\frac{R n-G}{1+\beta} \\
& H=\left[\frac{\beta}{(1+\beta)}\right](R n-G) \\
& \beta=\gamma \frac{\Delta T}{\lambda E} \\
& \beta=\frac{H}{\lambda E}
\end{aligned}
$$

em que,

$\beta$ - razão de Bowen;

$\mathrm{H}$ e $\lambda \mathrm{E}$ - fluxos de calor sensível e latente, respectivamente, $\mathrm{MJ} \mathrm{m}^{-2} \mathrm{dia}^{-1}$;

$\mathrm{Rn}$ - saldo de radiação, $\mathrm{MJ} \mathrm{m}^{-2} \mathrm{dia}^{-1}$;

$\mathrm{G}$ - fluxo de calor no solo, $\mathrm{MJ} \mathrm{m} \mathrm{mia}^{-1}$;

$\Delta \mathrm{T}$ - gradiente de temperatura do ar, ${ }^{\circ} \mathrm{C}$, e

$\gamma$ - constante psicrométrica, $0,062 \mathrm{kPa}^{\circ} \mathrm{C}^{-1}$.

\section{Instalação dos sistemas de balanço de radiação e medições micrometeorológicas}

Montaram-se duas torres micrometeorológicas, uma na parcela norte-sul e outra na lesteoeste. A diferença de temperatura entre os dois níveis $(0,2$ e 1,2 $\mathrm{m}$ acima da cultura) foi medida com termopares de cobre-constantan instalados em psicrômetros aspirados, construídos de acordo com MARIN et al. (2001). A voltagem diferencial gerada pelos termopares era produzida pela diferença de temperatura entre os níveis T1 - T2, para os termopares secos, e T1' - T2' para os molhados (Figura 1). A resolução das medidas de temperatura depende da resolução do sistema de aquisição de dados (datalogger), sendo nos equipamentos utilizados neste experimento, cerca de $0,006{ }^{\circ} \mathrm{C}$, com $0,1 \mu \mathrm{V}$ de ruído. 

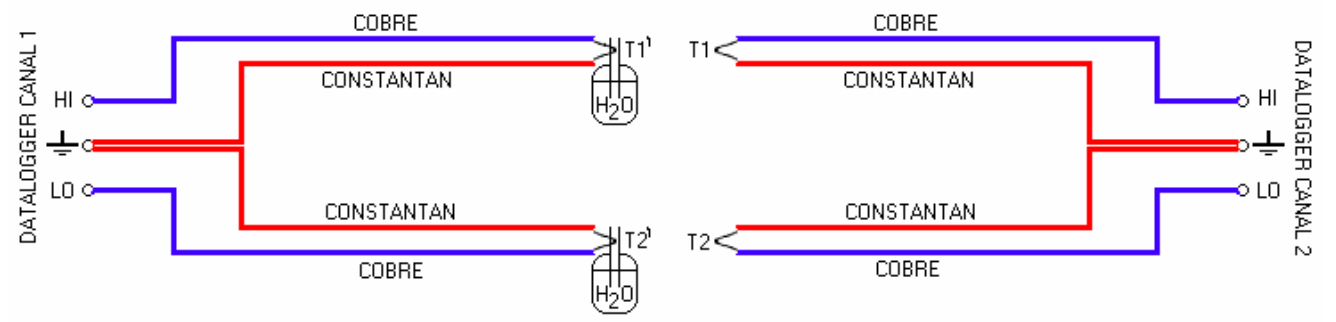

FIGURA 1. Configuração dos psicrômetros e esquema de ligação dos termopares de bulbo seco (T) e úmido ( $T^{\prime}$ ).

O saldo de radiação em cada parcela foi determinado com saldo-radiômetro (modelo Q7.1 Radiation and Energy Balance Systems, Washington, USA). Eles foram fixados em tubos de aço galvanizado de $25 \mathrm{~mm}$ de diâmetro e $2,5 \mathrm{~m}$ de altura, de modo que a cúpula do saldo-radiômetro ficava a $0,5 \mathrm{~m}$ acima da planta, para que sua visada também contemplasse a entrelinha. Geralmente, a técnica mais utilizada para determinar o fluxo de calor no solo $(G)$ é conhecida como método combinado. SILANS et al. (1997) não encontraram diferenças significativas entre os valores de evapotranspiração estimados com o método de balanço de energia pela razão de Bowen, quando utilizaram $G$ obtido pelo método combinado ou por placas enterradas na profundidade de $0,025 \mathrm{~m}$.

Neste experimento, $G$ foi obtido pelo valor médio dado apenas por duas placas (HFT3, Rebs, Seattle, Washington, EUA) enterradas próximas à superfície do solo, a 0,02 m, sendo uma a 0,2 m distante do tronco da planta, no lisímetro, e outra à mesma profundidade e distância, na planta, sob o saldo-radiômetro. Todos os sensores foram acoplados a dois sistemas de aquisição eletrônica de dados (modelo CR23X Campbell Scientific, Logan, Utah, EUA), programados para efetuar leituras a cada segundo e armazenar seus valores médios a cada 10 minutos.

\section{Critério para rejeição de dados inadequados do método RBBR}

Para rejeitar valores inconsistentes, incluindo aqueles fora dos limites da resolução instrumental, adotou-se análise física do método, conforme PEREZ et al. (1999). Segundo esses autores, a estimativa de $\lambda E$ e $H$ fornecida pelo método $R B B R$ deve ser consistente com a relação fluxo-gradiente, mas, em algumas vezes, as medidas dão sinais incorretos para esses fluxos. Dessa forma, as eqs.(1) e (3) podem ser arranjadas, resultando:

$$
\begin{aligned}
& \mathrm{Rn}=\left(1+\gamma \frac{\Delta \mathrm{T}}{\Delta \mathrm{e}}\right) \lambda \mathrm{E}+\mathrm{G} \\
& \frac{\Delta \mathrm{e}}{\lambda \mathrm{E}}=\gamma \frac{\Delta \mathrm{T}}{\mathrm{H}}=\frac{\Delta \mathrm{e}+\gamma \Delta \mathrm{T}}{\mathrm{Rn}-\mathrm{G}}>0
\end{aligned}
$$

em que,

$\Delta e$ - gradiente de pressão de vapor entre os níveis inferior e superior $(\mathrm{kPa})$.

A relação $\Delta \mathrm{e} / \Delta \mathrm{E}$, representada pela eq.(6), deve sempre ser maior que 0 , de acordo com a convenção de sinais (Figura 2). Os dados fornecidos pelo método $R B B R$ serão corretos quando atendem à desigualdade acima. Conseqüentemente, quando $R n-G>0, \Delta T>-\Delta e / \gamma$. Isso é, $\beta>-1$ se $\Delta e>0$, mas $\beta<-1$ se $\Delta e<0$. Quando $R n-G<0, \Delta T<-\Delta e / \gamma$. Isso é, $\beta<-1$ se $\Delta e>0$, mas $\beta>-1$ se $\Delta e<0$. As eqs.(1) e (2) mostram que apenas algumas combinações de valores de $H$ e $\lambda E$ são possíveis (Tabela 1). Quando $R n-G>0$, se $\beta>-1$, pelo princípio da conservação de energia, é deduzido que $\lambda E$ deve ser sempre positivo, visto que $H$ pode ser positivo ou negativo [eq.(2)], dependendo do sinal de $\beta$. Se $\beta<-1$, são possíveis apenas os casos $\lambda E>0$ e $H>0$. Quando $R n-G$ $<0$, se $\beta<-1$, são apenas possíveis os casos $\lambda E>0$ e $H<0$. Se $\beta>-1$, então $\lambda E$ será sempre negativo, visto que $H$ pode ser positivo ou negativo, dependendo do sinal de $\beta$. Se essas condições 
não forem satisfeitas (Tabela 1), o RBBR fornecerá um sentido incorreto de fluxo e os dados serão descartados. Isso apenas ocorre no início da manhã e ao entardecer, quando o fluxo de calor muda de sinal, durante a irrigação ou a precipitação, em que $\Delta e$ apresenta valores baixos (próximo do limite de resolução $\delta \Delta e$ ), e quando os valores de $R n-G$ são baixos. Para a análise dos dados e a exclusão de valores de $\beta$ inconsistentes, utilizaram-se as condições propostas por PEREZ et al. (1999), resumidas na Tabela 2.

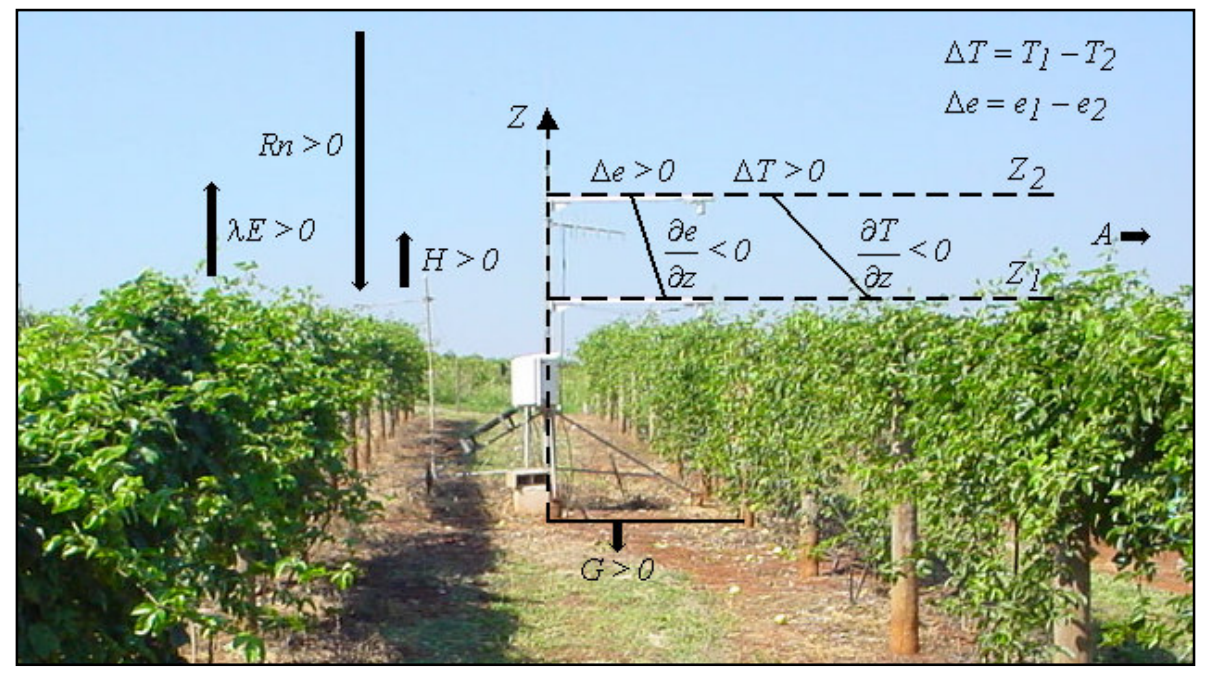

FIGURA 2. Representação dos fluxos de energia na interface entre o ar e a superfície vegetal, mostrando a convenção de sinais. $A$ - fluxo de energia advectiva para o volume de controle acima da superfície; $R n$ - saldo de radiação; $\lambda E$ e $H$ - fluxos de calor latente e sensível; $G$ - fluxo de calor no solo; $\Delta e$ e $\Delta T$ - diferença de pressão de vapor e temperatura entre os níveis inferior $\left(Z_{1}\right)$ e superior $\left(Z_{2}\right)$, e $\partial e / \partial z$ e $\partial T / \partial z$ - gradientes de pressão de vapor e temperatura.

TABELA 1. Limites de aceitabilidade dos valores obtidos pelo método $R B B R$ sob condições não-advectivas, de acordo com a eq.(6).

\begin{tabular}{cccc}
\hline Energia & $\begin{array}{c}\text { Gradiente de Pressão de } \\
\text { Vapor }\end{array}$ & $\begin{array}{c}\text { Razão de } \\
\text { Bowen }\end{array}$ & Fluxos de Calor \\
\hline$R n-G>0$ & $\Delta e>0$ & $\beta>-1$ & $\lambda E>0$ e $H \leq 0$ para $-1<\beta \leq 0$ ou $H>0$ \\
& $\Delta e<0$ & $\beta<-1$ & para $\beta>0$ \\
$R n-G<0$ & $\Delta e>0$ & $\beta>-1$ & $\lambda E<0$ e $H>0$ \\
& $\Delta e<0$ & $\beta<-1$ & $\lambda E>0$ e $H<0$ \\
& & $\lambda E<0$ e $H \geq 0$ para $-1<\beta \leq 0$ ou $H<0$ \\
& & para $\beta>0$ \\
\hline
\end{tabular}

$R n$ : saldo de radiação; $G$ : fluxo de calor no solo; $\Delta e$ : diferença de pressão de vapor entre os níveis superior e inferior; $\lambda E$ e $H$ - fluxos de calor latente e sensível, respectivamente.

TABELA 2. Resumo das classes de erros gerados pelo método RBBR (PEREZ et al., 1999).

\begin{tabular}{cl}
\hline Classe de Erro & Condição \\
\hline$A$ & $(R n-G)>0, \Delta e>0$ e $\beta<-1+|\varepsilon|$ \\
$B$ & $(R n-G)>0, \Delta e<0$ e $\beta>-1-|\varepsilon|$ \\
$C$ & $(R n-G)<0, \Delta e>0$ e $\beta>-1-|\varepsilon|$ \\
$D$ & $(R n-G)<0, \Delta e<0$ e $\beta<-1+|\varepsilon|$ \\
$E$ & Mudanças rápidas em $T$ e $e$ \\
\hline
\end{tabular}

$\overline{R n-G \text { : energia disponível; } \Delta e \text { : diferença de pressão de vapor entre o nível superior e inferior; } \beta \text { : razão de Bowen; } T \text { e }}$ $e$ : temperatura do ar e pressão de vapor; $\varepsilon$ : intervalo de erro que define o intervalo de exclusão dos valores da razão de Bowen; $\lambda \mathrm{E}$ e $H$ : fluxos de calor latente e sensível, respectivamente. 


\section{RESULTADOS E DISCUSSÃO}

Na Figura 3, apresenta-se o saldo de radiação médio diário $(R n)$ dos cultivos norte-sul e lesteoeste para os 14 meses de avaliação. As variações dos $R n$ durante o ano apresentaram valoreslimite de 5,28 a 13,52 $\mathrm{MJ} \mathrm{m}^{-2} \mathrm{dia}^{-1}$ para o cultivo com linhas norte-sul, e 6,18 a $14,66 \mathrm{MJ} \mathrm{m}^{-2} \mathrm{dia}^{-1}$ para o cultivo com linhas leste-oeste.

Nota-se, pelas curvas de $R n$, que as variações foram aproximadamente similares para os dois sistemas de cultivo, com pequena superioridade para o $R n$ do cultivo leste-oeste até meados de julho-2004, sendo superado após essa data pelo $R n$ do cultivo norte-sul. Essa inversão foi promovida, possivelmente, pela diminuição do fluxo de calor no solo do cultivo leste-oeste, nas estações de inverno e primavera.

Nessas estações, ocorreu sombreamento abaixo das plantas do cultivo leste-oeste na maior parte do período diurno e, conseqüentemente, menor incidência de radiação direta e menor armazenamento de calor no solo.

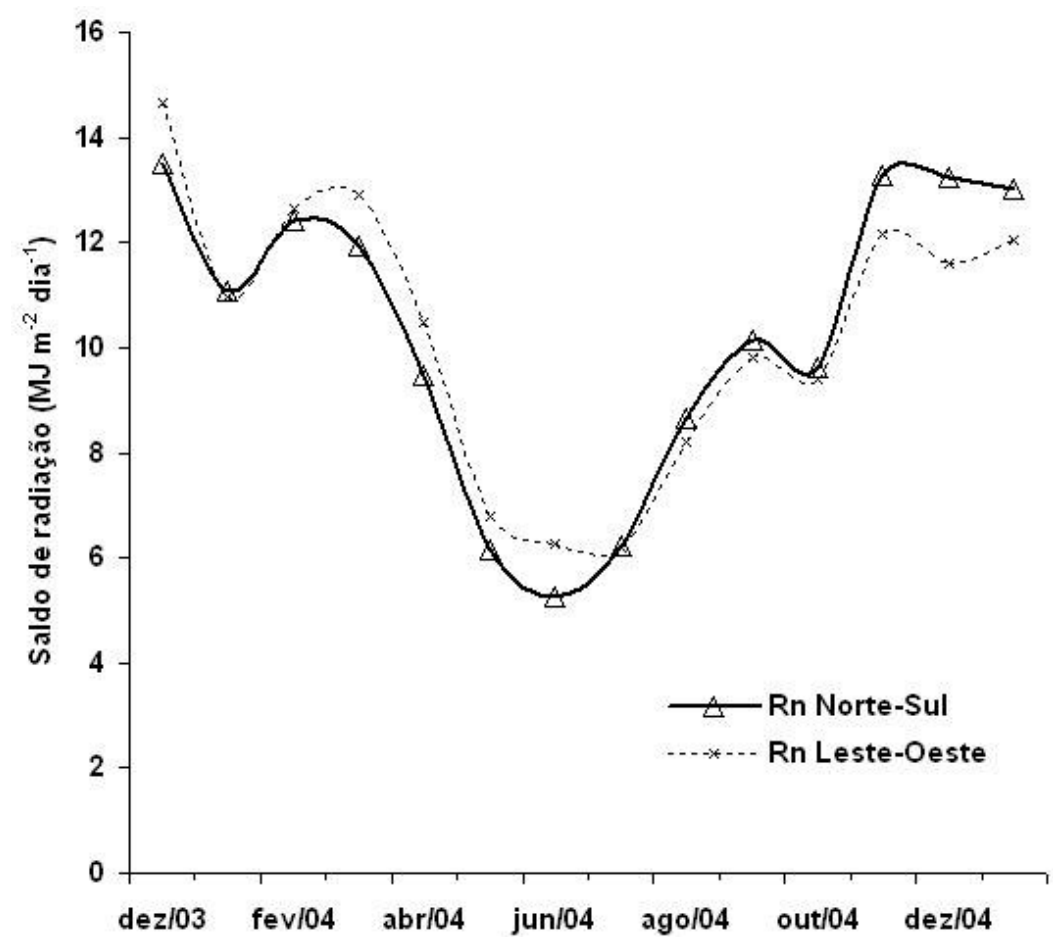

FIGURA 3. Evolução mensal do saldo de radiação $(R n)$ em culturas de maracujazeiro cultivado com linhas orientadas na direção norte-sul e leste-oeste, em Piracicaba - SP.

Realizando teste de média (teste t), foi possível determinar que não houve diferenças significativas entre os valores de $R n$ e $L E$ para os dois sistemas de cultivo durante os meses do ano (Tabela 3). Os fluxos médios mensais de calor no solo $(G)$ para o cultivo leste-oeste foram superiores e diferiram significativamente desde fevereiro até agosto, ou seja, final de verão, outono e inverno.

O movimento do Sol projetando-se sempre ao norte do local, nesse período (seguindo para o Equinócio de Outono e Solstício de Inverno), não promovia sombreamento no local do sensor de fluxo de calor no solo (sob a linha de cultivo), conseqüentemente, houve maior aquecimento do solo e maiores valores de $G$. No cultivo norte-sul, ao meio-dia (horário de maior incidência de valores de radiação), os sensores encontravam-se sombreados pelo renque da cultura, conseqüentemente, houve menores valores de $G$.

Relacionando os valores de saldo de radiação com a radiação global $(R g)$, obtiveram-se valores percentuais de $R n$ para os dois sistemas de cultivo. Nas curvas da Figura 4, observa-se que o percentual do $R n$ variou pouco com as estações do ano, com valor médio de 60,41 e 60,92\% para os cultivos norte-sul e leste-oeste, respectivamente. 
TABELA 3. Valores médios mensais, em MJ m${ }^{-2} \mathrm{dia}^{-1}$, do saldo de radiação $(R n)$; do fluxo de calor no solo $(G)$, da radiação global $(R g)$, do fluxo de calor latente $(L E)$ e do fluxo de calor sensível $(H)$, em Piracicaba - SP.

\begin{tabular}{|c|c|c|c|c|c|c|c|c|c|}
\hline \multirow{2}{*}{ Data } & \multicolumn{4}{|c|}{ norte-sul } & \multirow{2}{*}{$R g$} & \multicolumn{4}{|c|}{ leste-oeste } \\
\hline & $R n$ & $G$ & $L E$ & $H$ & & $R n$ & $G$ & $L E$ & $H$ \\
\hline dez./2003 & 13,52 & 1,97 & 10,90 & 1,58 & 21,56 & 14,66 & $-0,17$ & 9,98 & $3,98 *$ \\
\hline jan./2004 & 11,09 & 0,70 & 11,10 & $-0,71$ & 18,49 & 10,98 & 0,76 & 9,36 & $1,27 *$ \\
\hline fev./2004 & 12,42 & 0,03 & 13,55 & $-1,16$ & 20,36 & 12,66 & $0,63^{*}$ & 17,01 & 0,41 \\
\hline mar./2004 & 11,94 & $-0,22$ & 13,85 & $-1,69$ & 20,17 & 12,91 & $0,55^{*}$ & 13,43 & $0,96 *$ \\
\hline abr./2004 & 9,48 & $-0,29$ & 9,24 & 0,53 & 15,77 & 10,47 & $0,53 *$ & 10,06 & $-0,12$ \\
\hline maio/2004 & 6,14 & $-0,74$ & 6,24 & 0,63 & 11,17 & 6,81 & $-0,16^{*}$ & 7,15 & $-0,18$ \\
\hline jun./2004 & 5,28 & $-0,51$ & 5,76 & 0,08 & 10,10 & 6,27 & $0,18 *$ & 7,47 & $-1,06$ \\
\hline jul./2004 & 6,23 & $-0,43$ & 6,35 & 0,72 & 10,44 & 6,18 & $-0,10^{*}$ & 7,24 & $-0,21$ \\
\hline ago./2004 & 8,65 & $-0,40$ & 8,54 & 0,52 & 15,52 & 8,22 & $0,46^{*}$ & 8,03 & $-0,28$ \\
\hline set./2004 & 10,15 & 0,64 & 9,34 & 0,17 & 18,40 & 9,80 & 0,49 & 9,06 & 0,26 \\
\hline out./2004 & 9,63 & $-0,08$ & 9,49 & 0,22 & 14,89 & 9,40 & $-0,17$ & 10,47 & $-0,92$ \\
\hline nov./2004 & 13,29 & 0,36 & 12,94 & $-0,01$ & 20,15 & 12,14 & $-1,18$ & 14,42 & $-0,08$ \\
\hline dez./2004 & 13,22 & 0,26 & 14,31 & $-1,35$ & 19,79 & 11,60 & $-0,17$ & 11,82 & 0,07 \\
\hline jan./2005 & 13,01 & 0,00 & 12,80 & $0,31 *$ & 19,27 & 12,05 & 0,31 & 12,01 & $-0,23$ \\
\hline Média & 10,08 & 0,10 & 10,12 & $-0,04$ & 16,68 & 10,16 & 0,13 & 10,42 & 0,32 \\
\hline
\end{tabular}

* Diferença significativa a 5\% de probabilidade (teste $\mathrm{t}$ ).

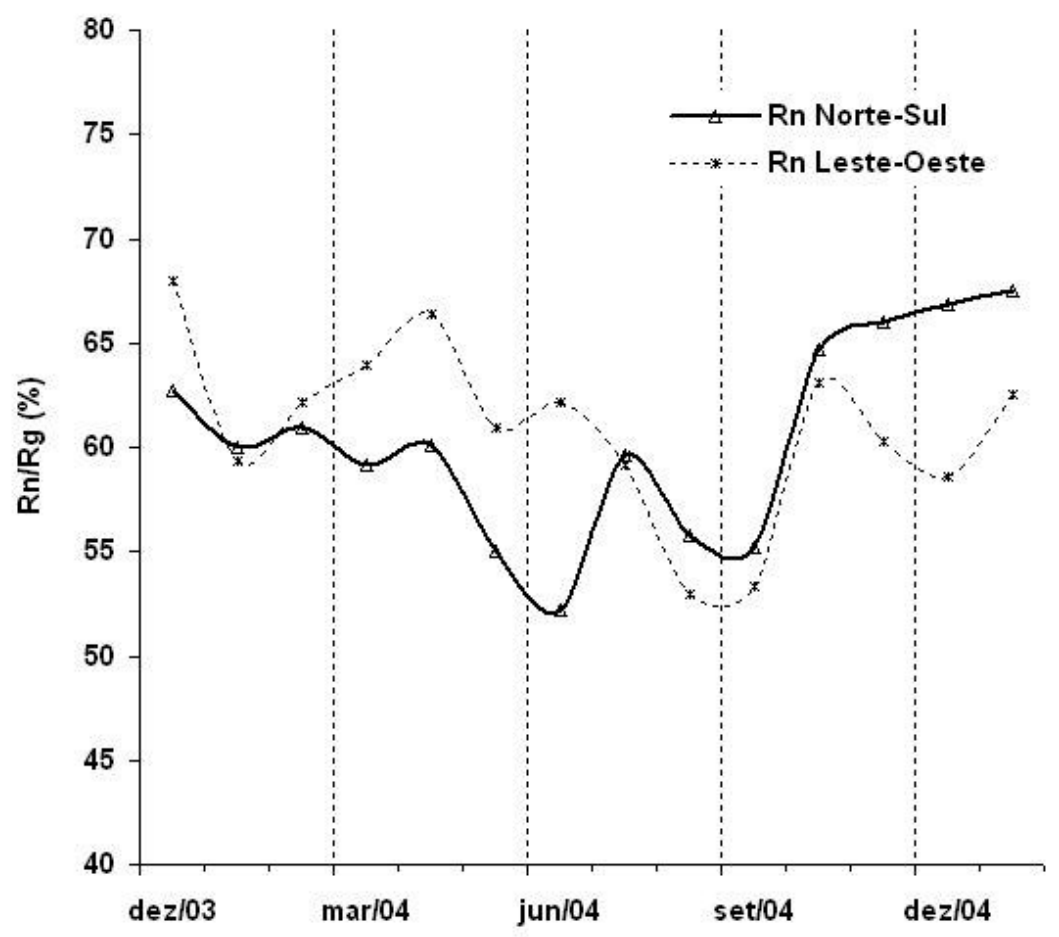

FIGURA 4. Percentuais de saldo de radiação $(R n)$ em relação à radiação global $(R g)$, nas estações do ano, sobre maracujazeiros cultivados em linhas norte-sul e leste-oeste, em Piracicaba - SP.

AZEVEDO et al. (1997), em Petrolina - PE, estudaram o saldo de radiação sobre a cultura de videira, observando aumento no percentual de $R n$ em relação à radiação global de 60 para $70 \%$ à medida que a cultura avançava do estádio inicial para o florescimento. Vale ressaltar que o cultivo estudado por esses autores foi conduzido em espaldeira horizontal, tipo caramanchão, que recobre totalmente a superfície do terreno, diferente da espaldeira vertical utilizada no cultivo de maracujazeiros. Para o espaçamento adotado ( $4 \mathrm{~m} \mathrm{x} 4 \mathrm{~m}$ ), o cultivo de maracujazeiro permitiu grande exposição do terreno (75 a 80\%). 
Os fluxos de calor latente $(L E)$ não diferiram para as orientações de cultivo durante a condução do experimento (Tabela 3). Em alguns meses, no início do estabelecimento do cultivo (dez./2003, jan./2004 e mar./2004), o fluxo de calor sensível $(H)$ do cultivo leste-oeste foi superior e diferiu estatisticamente do cultivo norte-sul. Essa situação ocorreu no verão quando $H$ do cultivo norte-sul atingiu valores mais negativos, especialmente após as chuvas ou irrigações, onde a superfície do solo se mantinha mais úmida, principalmente pela irrigação, devido ao sombreamento promovido pela cultura estabelecida pelas espaldeiras nessa direção.

Em função da latitude local e da variação da declinação solar no período, o cultivo leste-oeste promovia sombra sempre deslocada para o lado sul das linhas de cultivo (fora da região úmida do solo promovida pela irrigação). Essa condição proporcionou menor $L E$ e maior $H$ no cultivo lesteoeste, sendo o inverso observado no cultivo norte-sul.

Na Figura 5, representam-se as curvas percentuais da relação dos componentes $G, L E$ e $H$, com o saldo de radiação, para todos os meses do ano. Os percentuais médios anuais obtidos no cultivo norte-sul foram: $G / R n=-1,40 \%$; $L E / R n=100,67 \%$, e $H / R n=0,85 \%$. No cultivo lesteoeste, os percentuais foram: $G / R n=1,35 \% ; L E / R n=103,63 \%$, e $H / R n=0,91 \%$.

Nos dois sistemas de cultivo, a soma dos percentuais dos componentes do balanço de energia ultrapassou $100 \%$, o que indica sobreestimativa dos fluxos de calor latente e sensível, ou por influência de processos de advecção. Vários trabalhos com balanço de energia em cultivos irrigados, como o desta pesquisa, concluem que o maior percentual de radiação é transformado em fluxo de calor latente de evaporação (TEIXEIRA et al., 1997; CUNHA et al., 2002; PEZZOPANE \& PEDRO JÚNIOR, 2003).

Utilizando o critério de análise proposto por PEREZ et al. (1999), selecionaram-se os intervalos de amostragem e os valores de $\beta$ que se enquadravam nas classes de erro $(\varepsilon)$ apresentadas na Tabela 2.

Na Tabela 4, indicam-se o número total de intervalos amostrados realizado em cada cultivo, a percentagem total de dados que apresentaram algum tipo de erro e a percentagem dos dados falhos distribuídos por classe. Observa-se que, em média, 48,6\% dos intervalos amostrados apresentaram algum tipo de erro. Desse total, mais de $90 \%$ dos casos foram dados do período da noite, quando o $R n-G$ era positivo ou negativo com inversão do gradiente de pressão de vapor $(\Delta e)$. Os erros aconteceram, com maior freqüência, $45,6 \%$ para o cultivo norte-sul e $34 \%$ para o leste-oeste (casos tipo $B$ e $C$ ).

(A)

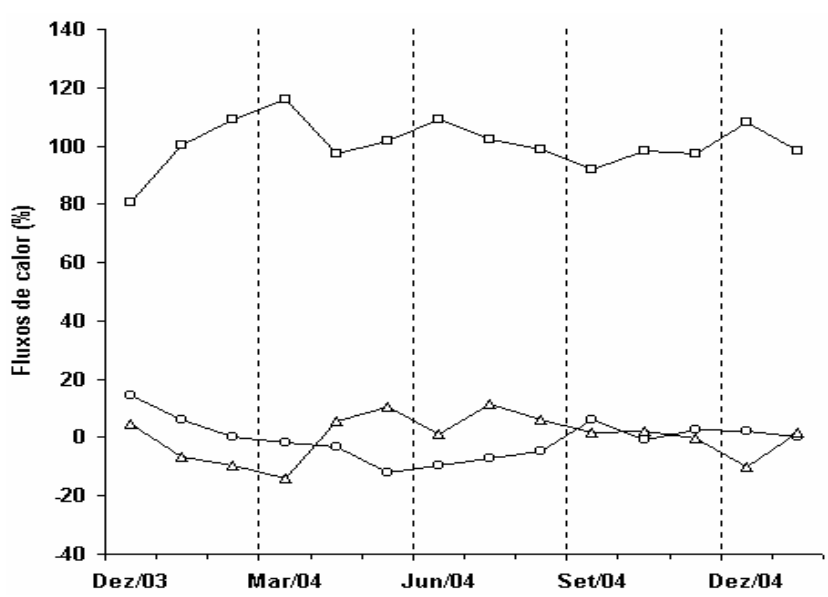

(B)

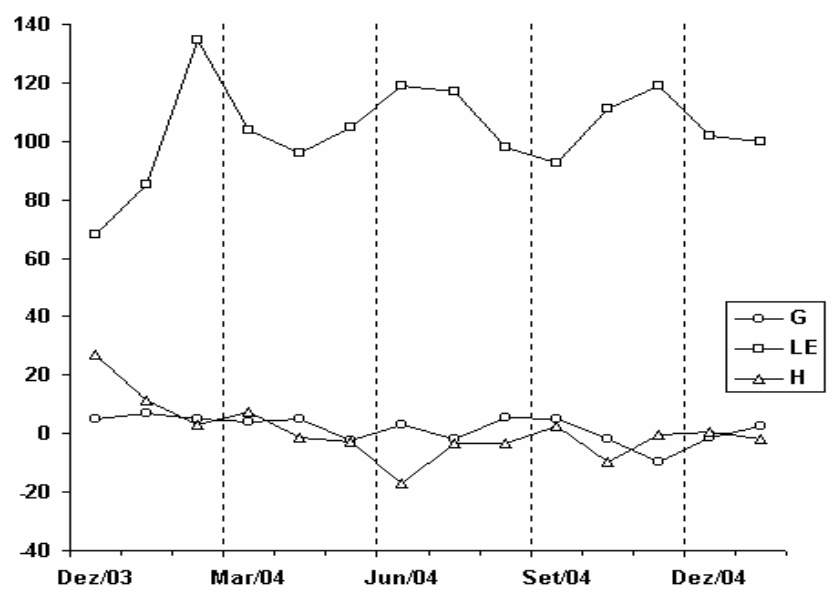

FIGURA 5. Curvas percentuais dos componentes de fluxo de calor latente $(L E)$, sensível $(H)$ e no solo $(G)$, em relação ao saldo de radiação, sobre maracujazeiros com linhas de cultivo norte-sul (A) e leste-oeste (B), em Piracicaba - SP. 
TABELA 4. Percentagem de casos em que os dados fornecidos pelo método $R B B R$ se enquadraram nos quatro tipos de erro (classes: $A, B, C$ e $D$ ), descritos na Tabela 1, para duas orientações de cultivo de maracujazeiros, em Piracicaba - SP.

\begin{tabular}{lccccc}
\hline \multirow{2}{*}{ Orientação (n ${ }^{-0}$ total de intervalos) } & $\mathcal{E}$-Total de Erros $(\%)$ & \multicolumn{4}{c}{ Tipo de Erro (\%) } \\
\cline { 3 - 6 } & & $A$ & $B$ & $C$ & $D$ \\
\hline norte-sul (59799) & 53,20 & 7,00 & 25,50 & 20,10 & 0,40 \\
leste-oeste (57693) & 44,07 & 9,00 & 15,00 & 19,00 & 0,70 \\
Média & 48,64 & 8,00 & 20,25 & 19,55 & 0,55 \\
\hline
\end{tabular}

$A, B, C$ e $D$ - Classes de erros gerados pelo método RBBR, segundo PEREZ et al. (1999), conforme Tabela 2.

A condição mais comum durante o dia é quando $R n-G$ e $\Delta e$ são maiores que zero. Porém, em condição de instabilidade atmosférica, se $\beta$ for inferior a $|\mathcal{E}|-1$, surgem casos associados ao erro tipo $A$. Esse erro corresponde aos períodos imediatamente após a chuva ou irrigação, com participação inferior a $10 \%$ dos casos. Os erros tipo $D$ tiveram participação muito baixa, inferiores a $1 \%$, e surgiram quando $R n-G$ foram menores que zero e o $|\mathcal{E}|-1$, maior que $\beta$.

PEREZ et al. (1999) encontraram valores médios de $15 ; 13 ; 12,5$ e $0,5 \%$ para os tipos de erro $A, B, C$ e $D$, respectivamente, em três localidades, no norte da Espanha, com clima semi-árido e dados coletados sobre cultivos de pequeno porte (centeio). Os autores também relataram que os horários mais suscetíveis a erros pelo método $R B B R$ são os de final de tarde, durante a noite e ao amanhecer, quando o saldo de radiação e o fluxo de calor no solo mudam de sinal, casos também observados nesta pesquisa.

Ao realizar teste de médias dos valores de evapotranspiração obtidos pelo método $R B B R$ entre sistemas de cultivos (norte-sul e leste-oeste), com e sem exclusão de intervalos de dados inconsistentes, foi possível identificar as épocas do ano em que o efeito "orientação de cultivo" interferia no processo de evapotranspiração (Tabela 5). Quando não se fez a exclusão dos intervalos de dados falhos fornecidos pelo método $R B B E$, não foi possível identificar diferenças entre as duas orientações de cultivo. Mas, realizando a análise e eliminando os intervalos inconsistentes, identificaram-se diferenças significativas entre as orientações de cultivo para os meses de dezembro-2003, janeiro, fevereiro, julho e agosto-2004.

TABELA 5. Evapotranspiração média mensal, em $\mathrm{mm} \mathrm{d}^{-1}$, obtida pelo método da Razão de Bowen-Balanço de Radiação $(R B B R)$, em maracujazeiros com linhas de cultivo orientadas norte-sul e leste-oeste, em Piracicaba - SP.

\begin{tabular}{|c|c|c|c|c|}
\hline & \multicolumn{2}{|c|}{ Intervalo Completo } & \multicolumn{2}{|c|}{ Intervalo com Dados Selecionados } \\
\hline & norte-sul & leste-oeste & norte-sul & leste-oeste \\
\hline dez./2003 & 5,41 & 4,24 & $5,81 *$ & 4,30 \\
\hline jan./2003 & 4,50 & 3,56 & $4,60 *$ & 3,45 \\
\hline fev./2004 & 5,42 & 4,08 & $5,24 *$ & 3,28 \\
\hline mar./2004 & 5,21 & 4,26 & 5,26 & 4,58 \\
\hline abr./2004 & 3,95 & 4,10 & 3,52 & 3,79 \\
\hline maio/2004 & 2,59 & 2,86 & 2,62 & 2,49 \\
\hline jun./2004 & 2,50 & 2,55 & 2,23 & 2,25 \\
\hline jul./2004 & 2,52 & 2,76 & $2,71 *$ & 1,96 \\
\hline ago./2004 & 3,55 & 3,35 & $3,88^{*}$ & 3,29 \\
\hline set./2004 & 3,80 & 3,69 & 4,09 & 3,90 \\
\hline out./2004 & 3,87 & 4,26 & 3,87 & 3,71 \\
\hline nov./2004 & 4,67 & 3,36 & 5,52 & 5,25 \\
\hline Média & 4,00 & 3,59 & 4,11 & 3,52 \\
\hline
\end{tabular}

* Diferenças significativas pelo teste $\mathrm{t}$, a $\%$ de probabilidade. 
Nas Figuras 6 e 7, observa-se a variação da evapotranspiração da cultura para os 14 meses de medida por meio de representação gráfica dos valores diários que geraram os valores médios da Tabela 5. Ajustaram-se os intervalos de dados com linha de tendência média móvel, resultante do agrupamento de 15 dados. Na Figura 6, utilizaram-se todos os intervalos gerados pelo método $R B B R$.

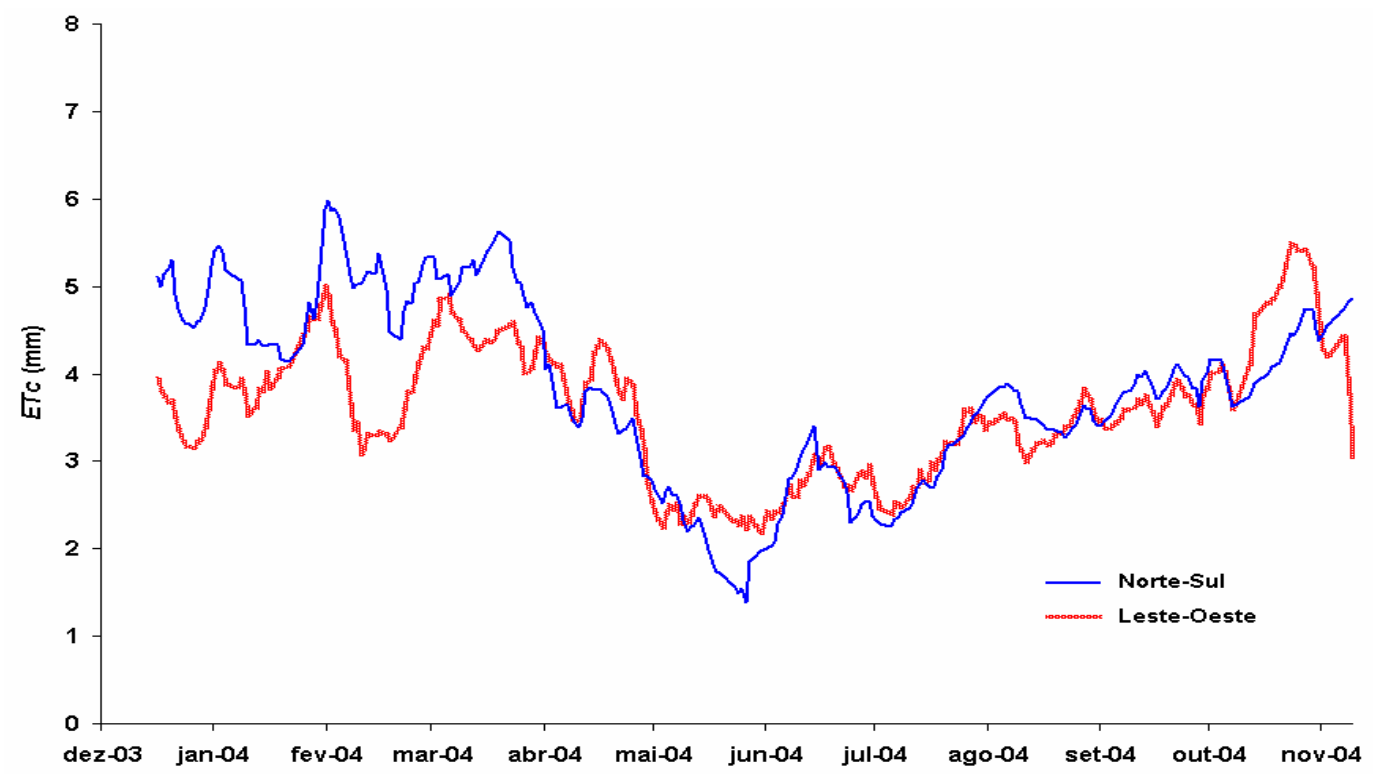

FIGURA 6. Curvas de evapotranspiração de maracujazeiros com linhas de cultivo orientadas na direção norte-sul e leste-oeste, obtidas pelo método da Razão de Bowen-Balanço de Radiação (intervalo completo), em Piracicaba - SP.

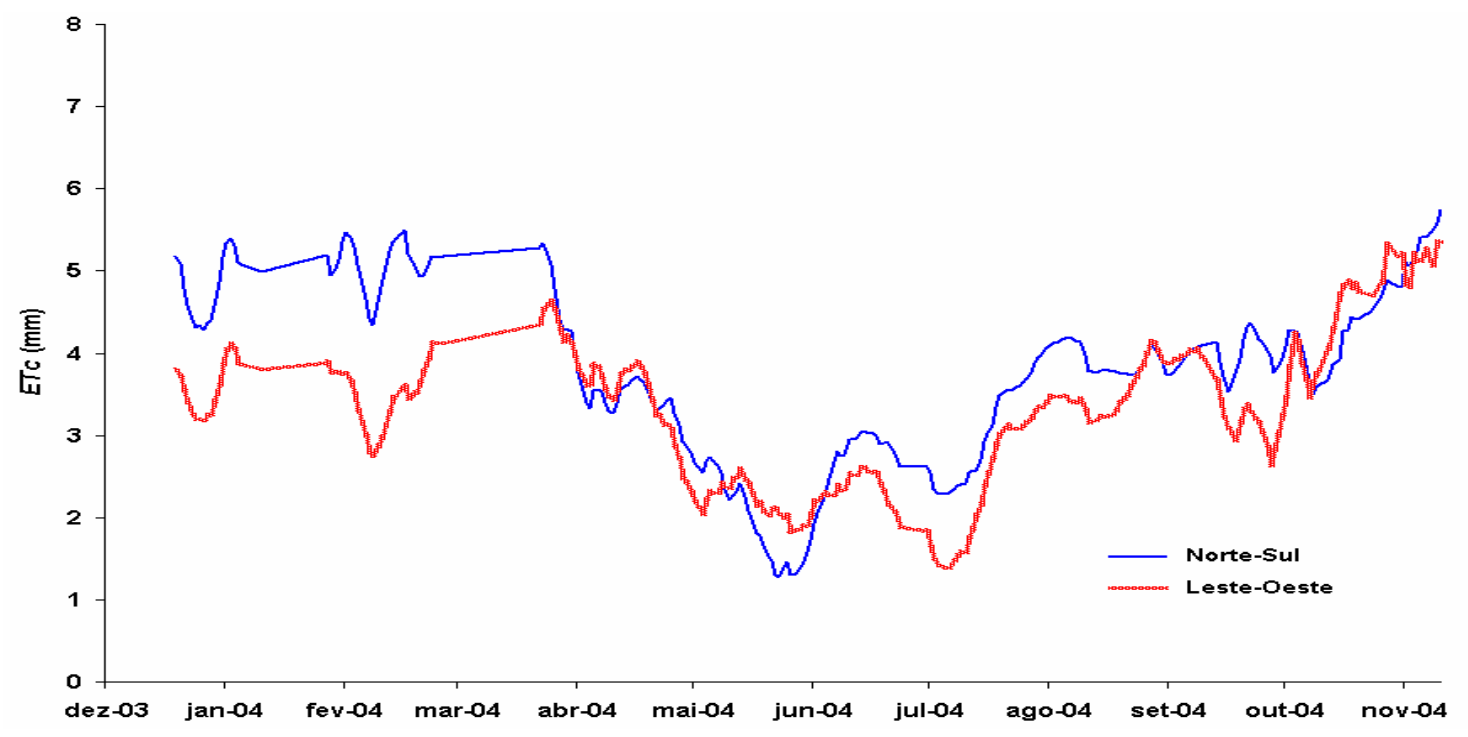

FIGURA 7. Curvas de evapotranspiração de maracujazeiros com linhas de cultivo orientadas na direção norte-sul e leste-oeste, obtidas pelo método da Razão de Bowen-Balanço de Radiação (intervalo com dados selecionados), em Piracicaba - SP.

A evapotranspiração média dos sistemas de cultivo, nas estações de verão, outono, inverno e primavera, foram de 5,19;2,08; 3,56 e 5,06 mm, respectivamente. A máxima evapotranspiração $(5,81 \mathrm{~mm})$ ocorreu no verão, para o cultivo norte-sul, e a mínima $(1,96 \mathrm{~mm})$ ocorreu no inverno, para o cultivo leste-oeste. Considerando-se todo o período analisado (14 meses), a evapotranspiração de maracujazeiros com linhas de cultivo orientadas na direção leste-oeste representou, em média, 87,25\% da evapotranspiração do cultivo norte-sul. Dessa maneira, o maior consumo verificado na orientação norte-sul, provavelmente, aconteceu porque, nessa orientação, 
houve maior interação com o vento, absorvendo mais energia da atmosfera, aumentando a evapotranspiração.

\section{CONCLUSÕES}

O saldo de radiação sobre o cultivo de maracujazeiros não foi influenciado pela orientação de plantio norte-sul ou leste-oeste. Seu valor foi equivalente a $60 \%$ da radiação global, durante as estações do ano, em Piracicaba - SP.

O componente de maior contribuição no balanço de energia foi o calor latente de evaporação, utilizando cerca de $100 \%$ da energia disponível.

O fluxo de calor sensível teve participação mínima no balanço de energia, enquanto o fluxo de calor latente de evaporação apresentou maior contribuição. Os maiores valores de fluxo de calor latente ocorrem imediatamente após as chuvas ou irrigações.

O sombreamento promovido pelo cultivo na direção leste-oeste influenciou significativamente no fluxo de calor no solo, nas estações de final de verão, outono e início do inverno.

A utilização do modelo de análise e exclusão de intervalos em que o método da Razão de Bowen falha, melhora a qualidade dos dados, permitindo verificar diferenciação na evapotranspiração da cultura no verão e inverno, quando o cultivo na direção norte-sul apresentou valores significativamente mais elevados em relação ao leste-oeste.

\section{REFERÊNCIAS}

AZEVEDO, P.V.; TEIXEIRA, A.H.C.; SILVA, B.B.; SORES, J.M.; SARAIVA, F.A.M. Avaliação da reflectância e do saldo de radiação sobre um cultivo de videira européia. Revista Brasileira de Agrometeorologia, Santa Maria, v.5, n.1, p.1-7, 1997.

BOWEN, I.S. The ratio of heat losses by conduction and by evaporation from any water surface, Physical Review, Ithaca, v.27, p.779-787, 1926.

CUNHA, A.R.; ESCOBEDO, J.F.; KLOSOWSKI, E.S. Estimativa do fluxo de calor latente pelo balanço de energia em cultivo protegido de pimentão. Pesquisa Agropecuária Brasileira, Brasília, v.37, n.6, p.735-43, 2002.

EMBRAPA. EMPRESA BRASILEIRA DE PESQUISA AGROPECUÁRIA. Centro Nacional de Pesquisa de Solos. Sistema brasileiro de classificação de solos. Rio de Janeiro, 1999. 412 p.

KUSTAS, W.P.; STANNARD, D.I.; ALLWINE, K.J. Variability in surface energy flux partitioning during Washita'92: resulting effects on Penman-Monteith and Priestley-Taylor parameters. Agricultural and Forest Meteorology, Amsterdan, v.82, n.1-4, p.171-93, 1996.

MARIN, F.R.; ANGELOCCI, L.R.; COELHO FILHO, M.A. Construção e avaliação de psicrômetro aspirado de termopar. Scientia Agricola, Piracicaba, v.58, n.4, p.839-44, 2001.

OMETTO, J. C. Bioclimatologia vegetal. São Paulo: Agronômica Ceres, 1981. 440 p.

ORTEGA-FARIAS, S.O.; CUENCA, R.H.; EK, M. Daytime variation of sensible heat flux estimated by the bulk aerodynamic meted over a grass canopy. Agricultural and Forest Meteorology, Amsterdan, v.81, n.1-2, p.131-43, 1996.

PEREIRA, A.R.; ANGELOCCI, L.R.; SENTELHAS, P.C. Agrometeorologia: fundamentos e aplicações práticas. Guaíba: Agropecuária, 2002. 478 p.

PEREZ, P.J.; CASTELLVI, F.; IBAÑEZ, M.; ROSELL, J.I. Assessment of reliability of Bowen ratio method for partitioning fluxes. Agricultural and Forest Meteorology, Amsterdan, v.97, n.3, p.141-50, 1999. 
PEZZOPANE, J.R.M.; PEDRO JÚNIOR, M.J. Balanço de energia em vinhedo de 'Niagara Rosada'. Bragantia, Campinas, v.62, n.1, p.155-61, 2003.

SILANS A.P.; MONTENY B.A.; LHOMME J.P. The correction of soil heat flux measurements to derive an accurate surface energy balance by the Bowen ratio method. Journal of Hydrology, Amsterdam, v.188-189, p.453-65, Feb., 1997.

SOUZA, J.S.I.; MELETTI, L.M.M. Maracujá: espécies, variedades, cultivo. Piracicaba: FEALQ, 1997. $179 \mathrm{p}$.

TEIXEIRA, A.H.C. Avaliação dos componentes do balanço de energia durante o primeiro ano de cultura da banana. Revista Brasileira de Engenharia Agrícola e Ambiental, Campina Grande, v.5, n.1, p.28-32, 2001.

TEIXEIRA, A.H.C.; AZEVEDO, P.V.; SILVA, B.B.; SOARES, J.M. Balanço de energia na cultura da videira, cv. 'Itália'. Revista Brasileira de Agrometeorologia, Santa Maria, v.5, n.2, p.137-41, 1997.

UNLAND, H.E.; HOUSER, P.R.; SHUTTLEWORTH, W.J.; YANG, Z.L. Surface fluxe measurement and modeling at a semi-arid Sonoran Desert site. Agricultural and Forest Meteorology, Amsterdan, v.82, n.1-4, p.119-53, 1996. 